\title{
TV/Series
}

15 | 2019

La Sérialité en question(s)

\section{La sérialité en question(s)}

Introduction

Florent Favard et Hélène Machinal

\section{CpenEdition}

Journals

Édition électronique

URL : http://journals.openedition.org/tvseries/3388

DOI : 10.4000/tvseries.3388

ISSN : 2266-0909

Éditeur

GRIC - Groupe de recherche Identités et Cultures

Référence électronique

Florent Favard et Hélène Machinal, «La sérialité en question(s) », TV/Series [En ligne], 15 | 2019, mis en ligne le 16 juillet 2019, consulté le 24 septembre 2020. URL : http://journals.openedition.org/tvseries/ 3388 ; DOI : https://doi.org/10.4000/tvseries.3388

Ce document a été généré automatiquement le 24 septembre 2020.

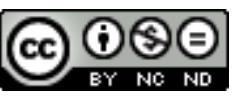

TV/Series est mis à disposition selon les termes de la licence Creative Commons Attribution - Pas d'Utilisation Commerciale - Pas de Modification 4.0 International. 


\title{
La sérialité en question(s)
}

\author{
Introduction
}

\author{
Florent Favard et Hélène Machinal
}

1 Les séries télévisées contemporaines sont aujourd'hui une forme audiovisuelle populaire et reconnue par la critique universitaire, loin de leur image d'antan, celle du programme télévisuel fatalement "inférieur» au cinéma et désespérément « répétitif ». Ces deux aspects, soit la hiérarchisation et la répétition, ont été abordés dès l'article fondateur d'Umberto Eco sur la répétition et la variation (1994). La question de la fiction "industrielle" (St Beuve), que l'on oppose à des formes de création dites plus «nobles» et supposément détachées de tout contexte de production, peut se repérer bien en amont des révolutions industrielles. Le théâtre de Shakespeare, le roman à sensation, le récit policier relèveraient encore d'une littérature «populaire» ou de la «paralittérature». Ces considérations, nous le verrons, ne sont pas à balayer d'un revers de la main.

2 Au sein de l'université, et notamment dans de nombreuses disciplines qui souhaitent se pencher sur cet objet d'étude (études médiatiques, études culturelles, études en arts visuels, sociologie, etc.), persiste encore l'idée qu'il n'existe pas de cadre théorique pour aborder la forme sérielle d'un point de vue esthétique. Sociologie, sciences de l'information et de la communication, études anglophones ont su y apporter et adapter leurs bagages conceptuels; mais lorsque vient l'heure de décrire et d'analyser la nature de l'objet, du médium, plane encore la comparaison avec l'analyse filmique. Cette comparaison est maintenue à flots par les déclarations récurrentes de réalisateur.ice's se lançant dans l'expérience sérielle en l'envisageant comme un film de très longue durée; là encore, il convient de ne pas ignorer l'assimilation du dispositif télévisuel (qu'est-ce que le télévisuel à l'heure du numérique, demande par exemple Amanda Lotz) au dispositif cinématographique, surtout lorsque les unités discrètes que sont les épisodes se retrouvent agglomérées, indifférenciées, dans le flot d'une structure d'ordre supérieur, la saison, voire la série, au travers de pratiques de visionnage (bingewatching) comme de diffusion (les livraisons par saisons sur Netflix).

3 Lorsqu'elle est esthétique, l'analyse des séries est aussi dominée, jusque dans ce numéro, par des modèles narratologiques: une prédominance à double tranchant, 
puisque ces modèles peinent encore à trouver pied au milieu d'une effervescence de notions et concepts parfois contradictoires, quand ils ne désignent pas des mécanismes similaires sous des dénominations variables, ne serait-ce qu'en France. L'analyse des séries télévisées se constitue petit à petit comme un champ transdisciplinaire, et se nourrit de ces complexités et contradictions.

La série télévisée, malgré toutes ses évolutions, reste (pour le moment...) caractérisée par sa division en épisodes, par sa sérialité ; celle-ci a été explorée dans le détail, notamment depuis le début des années 2000 et la montée de ce que Jason Mittell nomme la complexité narrative (2015) : ce mode narratif est caractérisé entre autres par la fusion entre les rythmes de la série épisodique, aux occurrences indépendantes, et du feuilleton doté d'un continuum narratif. La série feuilletonnante, standard narratif de ces trois dernières décennies, continue toutefois d'évoluer; on aurait aussi tort de croire que les hybridations des années 1980 à 2000 ont été décrites de manière exhaustive: de même que la série Lost ( $\mathrm{BBC}, 2004-2010)$, la recherche sur les séries télévisées continue de se nourrir de flashbacks comme de flashforwards.

5 En France, longtemps portée aux nues, la notion de formule développée par Jean-Pierre Esquenazi (2011) - ces invariants et "points nodaux» qui équilibrent répétition et variation - est remise en question, y compris par le principal intéressé ; même constat pour le concept fluide de "matrice ", qu'il se confonde plus ou moins avec la formule chez Stéphane Benassi (2000), ou vienne la contrebalancer chez Guillaume Soulez (2011).

6 La théorie des mondes possibles appliquée à la fiction connaît aussi un renouveau au travers de l'analyse de séries qui possèdent souvent des extensions transmédiatiques et s'ouvrent à une écriture des possibles : Nathalie Maroun (2009) valorise son importance pour analyser des mondes fictionnels en déploiement perpétuel; Sarah Hatchuel pousse plus loin le raisonnement avec la notion de "plis hypothétiques ", des possibles narratifs explorés dans les rêves ou les réalités alternatives (2015) ; Florent Favard y voit le creuset de la notion de «mythologie », notamment dans les séries de sciencefiction (2018).

7 La complexité narrative a eu, et a encore, un riche succès, la réflexion longue de plus d'une décennie de Jason Mittell ayant été complétée par des approfondissements sur la construction du personnage sériel (Pearson, 2007), sur la temporalité (Booth, 2012), la téléologie du récit sériel (Favard, 2019) et sa déformulation finale (Lifschutz, 2018), ou encore la transmédialité (Kustritz, 2014 ; Cornillon, 2018). Des thèses récemment soutenues témoignent d'un champ d'études bouillonnant et en perpétuelle évolution, par exemple en France, qu'il s'agisse de l'exploration du « tissage narratif » par Hélène Breda (2015), l'expérience du récit sériel par Anaïs Goudmand (2018), ou le format de la websérie par Deborah Gay (2019). Il existe sans nul doute de nombreux travaux en cours dans d'autres pays - Allemagne, Espagne, Italie, Angleterre, Etats-Unis... - qui abordent les problématiques variées véhiculées par la sérialité télévisuelle.

8 Se situant dans le prolongement d'autres numéros (que l'on pense par exemple au n 3 de Mise au point publié en 2011, ou au $n^{\circ} 14$ de Belphégor, sorti en 2016), ce dossier entend offrir une "nouvelle saison" aux questionnements sur la sérialité, en se focalisant sur les séries télévisées contemporaines. L'objectif de ce numéro de TV/Series est d'aborder la sérialité, qualité fondamentale des séries télévisées, dans un cadre théorique et conceptuel, sous des angles narratologiques, et plus largement esthétiques et sémiotiques. Les articles recueillis dans ce numéro attestent du renouveau de la 
critique sérielle mais n'entendent pas couvrir l'ensemble de la réflexion que la sérialité induit. Les analyses retenues s'inscrivent dans un chantier à long terme qui doit, en outre, tenir compte de l'évolution constante de la forme sérielle, qui ne peut être dissociée de celle des modes de production et de diffusion dont on a pu récemment constater qu'ils n'étaient pas en reste lorsqu'il s'agit d'offrir de nouveaux types d'accès aux fictions.

Divisé en trois parties, ce dossier commence par revenir sur l'évolution de la complexité narrative et les nouvelles pistes de lecture que l'on peut extraire des hybridations passées et présentes à l'œuvre dans les séries télévisées. Claire Cornillon ouvre la saison avec une étude de cas centrée sur Ally McBeal (Fox, 1997-2002), dans laquelle elle prolonge des réflexions en cours sur les formes hybrides des années 1990 qu'elle nomme " semi-feuilletonnantes » : elle avance ainsi que l'aspect procédural, très épisodique, de la série (le client de la semaine dans un cabinet d'avocats) n'empêche en rien le déploiement d'une trame feuilletonnante, pas parce que cette dernière s'accommode de la répétition, mais parce qu'elle y trouve sa structure même, fondée sur un processus d'empathie sans cesse renouvelé et une ouverture sur les vies des personnages récurrents comme des clients d'un jour. Elaine Després poursuit dans une veine similaire, en s'interrogeant sur l'impact de la généricité sur la gestion de la sérialité dans deux séries des années 1990, The X-Files (Fox, 1993-2002) et Buffy the Vampire Slayer (The WB > UPN, 1997-2003). Elle s'intéresse particulièrement au statut élusif de l'épisode autonome, qui profite d'une formule rôdée mise en place au fil des saisons pour mieux s'en écarter via un manque ou une rupture, proposant des espaces de liberté inattendus pour les scénaristes comme pour les personnages: boucles temporelles, changements d'identités, pastiches, métalepses, sont autant de cascades narratives et transtextuelles à même de se distinguer de la série pour mieux la caractériser. Ancré dans le présent immédiat, Benjamin Campion clôt cette première partie par le constat inverse : sur Netflix, l'épisode perd cette capacité à se comporter en unité discrète, noyé dans un flux de diffusion que la plateforme de SVoD coordonne à un type de visionnage encouragé, voire presque forcé: le binge-watching, ou visionnage en marathon. Plus largement, à l'aune des dernières évolutions du récit sériel, il s'interroge sur les renoncements tacites à la sérialité mis en place par Netflix et consorts : renégociation du rêve collectif, de la discussion permise par l'attente du prochain épisode ; disparition de l'épisode, même - et surtout - du pilote ; permission de passer outre le paratexte répétitif et pourtant riche de variations que sont les résumés des épisodes précédents et génériques de début et fin. Au fil de ces trois contributions, c'est toute la tension, l'hybridation et l'équilibre entre épisodique et feuilletonnant qui se retrouve questionnée dans son rapport au récit mais aussi à sa réception.

10 La seconde partie de ce dossier nous amène à voyager, et ouvre la voie à des analyses qui, si elles restent marquées par un cadre narratif, s'intéressent à la dimension spatiale des récits proposés par les séries contemporaines. Hélène Breda, dont la thèse sur le tissage narratif fait depuis quelques années son chemin, poursuit ses explorations en s'intéressant à une nouvelle analogie textile, celle de l'« étoffement » mesurable dans les adaptations sérielles de romans, étoffement qui ne mesure pas seulement en fonction du nombre de personnages, mais aussi et surtout de leurs riches interactions marquées par des polarisations sans cesse renégociées. La série de science-fiction The Man in the High Castle (Amazon, 2015-) lui sert de cas d'étude ; ainsi elle ouvre le champ de son appareil théorique et méthodologique en proposant d'analyser des œuvres 
déployant des mondes à la cosmologie plurielle au sens de Marie-Laure Ryan, c'est-àdire eux-mêmes subdivisés en plusieurs réalités alternatives. Diane Bénédic-Meyer arpente une route parallèle, se focalisant non pas sur des réalités alternatives, mais sur des temporalités disjointes, au travers de l'analyse de la série Outlander (Starz, 2014-); reprenant le concept de tissage narratif, elle s'interroge sur le tissage émotionnel au féminin des aventures de l'héroïne principale, et sur le gap, le fossé qui la sépare de son amant vivant dans l'Écosse jacobite du XVIII ${ }^{\mathrm{e}}$ siècle. Elle déploie ce tissage jusque dans la sphère de la réception, montrant combien les vies des personnages et de leurs interprètes s'entremêlent aujourd'hui. Toujours focalisée sur la spatialité des séries télévisées, mais dans un tout autre registre, Sophie Lorgeré propose «d'appréhender les séries télévisées à l'aune de leur composition spatiale » en prêtant une attention toute particulière à l'évolution du rapport entre espace tangible et espace mental au fil des épisodes de la série Hannibal (NBC, 2013-2015), pour montrer comment ces espaces, et leur procédé de représentation évolutif, traduisent et annoncent le brouillage des frontières entre le docteur Lecter et le profiler Will Graham, entre visions hallucinatoires, souvenirs troubles et palais mémoriels.

11 La troisième et dernière partie de ce dossier, nous l'avons réservée aux contributions cherchant à échapper au prisme narratologique qui domine encore l'analyse des séries télévisées. Elle commence par la proposition de Chloé Delaporte d'appliquer la sémiopragmatique de Roger Odin aux shortcoms françaises, et notamment à Scènes de ménages (M6, 2009-), Nos chers voisins (TF1, 2012-2017) et Parents mode d'emploi (France 2, 2013-). Elle cherche à démontrer combien ces formats courts ne peuvent être envisagés de la même manière que des séries à épisodes plus longs, notamment parce que leurs procédés sériels privilégient la spectacularisation sur la diégétisation, donnant à voir, $\mathrm{du}$ fait de leurs contraintes techniques et économiques, des espaces clos régulièrement rythmés par des transitions animées qui ne permettent pas « d'effacer le support » au sens d'Odin. Ces pistes fascinantes une fois élaborées, le dossier se conclut par deux articles-sommes, écrits à plusieurs mains, qui achèvent non pas d'invalider le prisme narratologique, mais de questionner sa prédominance. Marta Boni et Mireille Berton interrogent notre capacité à explorer des objets aussi complexes (au sens de Morin) et fluides que les séries télévisées, arguant que leur quantité, leur instabilité et leur complexité autorisent d'autres approches, notamment une approche spatiale fondée cette fois non pas sur l'espace représenté, mais sur l'espace de diffusion et de réception, dépassant " la sphère de l'intertexte pour cerner le contexte ", qu'il soit économique ou géographique par exemple. Elles nous invitent ainsi à envisager la série télévisée " comme une sémiosphère ", proposant des pistes focalisées sur l'analyse de traces électroniques. Écrit à quatorze mains comme le serait une série américaine, la contribution de l'association Saute-Requin, basée à Rennes 2, fonctionne comme les derniers épisodes de Lost, revenant loin dans le passé pour mieux interroger le présent et le futur des séries télévisées : c'est en remontant à l'origine du terme « série » et en analysant ses différentes acceptions dans le monde de l'art au fil du temps que le collectif entend proposer des pistes alternatives au prisme narratologique, notamment en se recentrant sur la plasticité du médium et ses capacité expressives sans cesse renouvelées.

$12 \mathrm{Au}$ terme de ce dossier, nous n'entendons pas épuiser les réflexions sur la sérialité, mais bien apporter notre pierre à un édifice en perpétuelle (re)construction, au travers de nouveaux questionnements sur la complexité narrative au fil des décennies, sur les approches spatiales et sur les limites des analyses narratologiques. On le sait, les séries, 
et la sérialité dans son ensemble, n'ont pas fini de faire parler d'elles, et les prochains épisodes s'annoncent tout aussi passionnants.

\section{BIBLIOGRAPHIE}

ALLRATH, Gaby (dir.), GYMNICH (dir.), Narrative Strategies in Television Series, Houndmills, New York, Palgrave MacMillan, 2005

AMES, Melissa (dir.), Time in TV Narrative: Exploring Temporality in Twenty-First-Century

Programming, Jackson, University Press of Mississippi, 2012

BENASSI, Stéphane, Séries et feuilletons T.V. : pour une typologie des fictions télévisuelles, Liège, Éditions du CEFAL, 2000

BENNETT, Tara, Showrunners: The Art of Running a TV Show, Londres, Titan Books, 2014

BREDA, Hélène, Le tissage narratif dans les séries télévisées américaines contemporaines, thèse de doctorat, dir. Guillaume Soulez, Paris III, 2015

BOOTH, Paul, « Memories, temporalities, fictions: temporal displacement in contemporary television », Television \& New media, 12(4), 2011

COLONNA, Vincent, L'Art des séries télé 1 : L'Appel du happy end, Paris, Payot, coll. « Petite bibliothèque », 2015 (réédition poche)

CORNILLON, Claire, Sérialité et transmédialité. Infinis des fictions contemporaines, Paris, Honoré Champion, 2018

DELEUZE, Gilles, Différence et répétition, Paris, PUF, 1968

ECO, Umberto, «Innovation et répétition : entre esthétique moderne et post-moderne », Réseaux, $\mathrm{n}^{\circ} 68,1994$, p. $1-18$

ESQUENAZI, Jean-Pierre, Les Séries télévisées : L'Avenir du cinéma ?, Paris, Armand Colin, 2010

FAVARD, Florent, Le récit dans les séries de Science-fiction de Star Trek à X-files, Paris, Armand Colin, 2018

FAVARD, Florent, Écrire une série TV. La Promesse d'un dénouement, Tours, PUFR, coll. « Sérial », 2019

GAY, Deborah, Le Genre de l'innovation, la production de la différence : dans les coulisses d'une websérie, thèse de doctorat, dir. Marlène Coulomb, Toulouse 2, 2019

GOUDMAND, Anaïs, Récits en partage. Expériences de la sérialité narrative en culture médiatique, dir. Jean-Marie Schaeffer et Raphaël Baroni, Paris Sciences et Lettres, 2018

HATCHUEL, Sarah, Rêves et séries américaines, Paris, Rouge Profond, 2016

KUSTRITZ, Anne, "Seriality and Transmediality in the Fan Multiverse", TV/Series, \#6, 2014

LAVERY, David, « Lost and Long-Term Television Narrative », in HARRIGAN, Pat (dir.), WARDRIPFRUIN, Noah (dir.), Third Person: Authoring and Exploring Vast Narratives, Cambridge, Londres, MIT Press, 2009 
LIFSCHUTZ, Vladimir, This is the end. Finir une série TV, Tours, PUFR, coll. « Sérial », 2018

LOTZ, Amanda D., The Television Will Be Revolutionized, New York, New York University Press, 2007

MAROUN, Natalie, Continuité narrative et jeux des possibles dans l'écriture scénaristique des séries télévisuelles, thèse de doctorat, dir. Marc Escola, Charif Majdalani, Paris VIII, 2009

MICHLIN, Monica, « More, More, More: Contemporary American TV series and the attractions and challenges of serialization as ongoing narrative", Mise au point, $n^{\circ} 3,2011$

MITTELL, Jason, Complex TV: The Poetics of Contemporary Television Storytelling, New York, Londres, New York University Press, 2015

NEWMAN, Michael, « From beats to arcs: Toward a poetic of television narrative », The Velvet Light Trap, $\mathrm{n}^{\circ}$ 58, 2006

ÖRNEBRING, Henrik, "The Show Must Go On... And On: Narrative and Seriality in Alias ", in ABBOTT, Stacey (dir.), BROWN, Simon (dir.), Investigating Alias: Secrets and Spies, Londres, New York, I.B. Tauris, 2007

PEARSON, Roberta, « Anatomising Gilbert Grissom: The Structure and Function of the Televisual Character ", in ALLEN, Michael, Reading CSI: Crime TV Under the Microscope, Londres, I.B. Tauris, 2007

SOULEZ, Guillaume, «La double répétition : structure et matrice des séries télévisées », Mise au point, $\mathrm{n}^{\circ} 3,2011$ 